\title{
Theoretical Aspects of Sustainable Development (SD) and Industrial Energy (IE)
}

\author{
${ }^{1}$ Sim Sze Yin and ${ }^{2}$ Yoni Danieli \\ ${ }^{1,2}$ Department of Computer Science and Engineering Yonsei University, Seoul, Korea \\ 1'simszeyin75@aol.com
}

\begin{abstract}
ArticleInfo
Journal of Machine and Computing (http://anapub.co.ke/journals/jmc/jmc.html)

Doi : https://doi.org/10.53759/7669/jmc202101009

Received 20 December 2020; Revised form 26 January 2021; Accepted 25 March 2021; Available online 05 April 2021.

(C2021 The Authors. Published by AnaPub Publications.

This is an open access article under the CC BY-NC-ND license. (http://creativecommons.org/licenses/by-nc-nd/4.0/)

Abstract - The theoretical assumption of Sustainable Development (SD) is gradually beginning to be applied in various industrial segments. One on the segments is industrial energy, which is significantly utilized and intuitively comprehended even though there is no full explanation of its phenomena. The main aim of this research contribution is to discuss the theoretical assumptions of SD and Industrial Energy (IE). This paper considers critically all the issues of permanence since it incorporates the environmental and social needs for ecological development. In addition, overall characterized, the major segments of these theoretical assumptions are based on critical analysis of the literature source.
\end{abstract}

Keyword - Sustainable Development (SD), Sustainable Energy (SE), Industrial Energy (IE)

\section{INTRODUCTION}

Global predictions on the consumption of industrial energy projects more development and demand of energy, power and temperature. The predictions incorporate valuable data on the futuristic structures of industrial Energy (IE). More domination of fossil fuels has been projected up to 2050, which represents the major influence of humans on the ecosystem, which is even projected to increase further. This in turn alters the concerns on retainability of the stabilized access of power and energy reserves and the potential reduction of the speed of climatic change. These concerns concerning the durability of the present energy policies amount to the advancing interests in the theoretical assumptions of Sustainable Development (SD).

Stable accessibility to power, energy and temperature is considered a fundamental element in the enhancement of civilization. Nonetheless, in literature and policy evaluation, it is challenging to discern an intuitively clear explanation of how IE sector develop strategies modelling based on the concepts of SD. The explanations and assumptions are drawn to two aspects i.e. the enhancement of renewable sources and efficiency of IE and its implementation processes. The main aim of this contribution is to characterize and define the theoretical assumptions of SD and IE. The first impression created by this research is much broader since the prevailing concern of sustainability represents the generate IE and the consumption of power and temperature. Evaluating a balanced strategy to IE has to be considered on not only the issue of $\mathrm{SD}$, but also the incorporation of environmental and social needs for economic enhancement.

Even though the introduction of SD can be dated back to the 1970s, is in the power of WCED (World Commission on Environmental Development) that the term (sustainable development) was explained and coined [1]. SD was defined as the enhancement that meets the requirements of the status of IE without altering the capacity of the future generation in accomplishing their own energy, power and temperature needs. One of the definitive moments of sustainability was based on the earth summit at Rio de Janeiro in 1992, which led to the agreements of launching the procedures of developing SD goals and objectives. These goals and objectives were utilized as a tool for pursuing coherent and focused actions on SD.

However, why is SD fundamental for rural enhancement? Industrial poverty is a predominant rural issue with the wider portion of poor consumers found in rural settlements. According to researchers, $76 \%$ of the globe's poor reside in rural environments, which is more than the $58 \%$ standard and overall portion of rural dwellers. The concept of poverty significantly limits the quality and quantity of industrial products that can be consumed [2]. The people working in developing countries normally generate about $\$ 2$ in single data, which is significantly lower than what is obtained in other developed countries.

According to the assumptions and declarations at Rio, SD and IE are cornerstones for industrial development. From Rio, twenty-seven principles (Ps) were set to promote the assumptions such as centrality of man to the SD concerns as P1. Poverty primacy and its eradication was set as P5; while the significance of the ecology for the present and future generation and its footing with the industrial development P3 and P4. The particular consideration provided to developing 
nations was P6. The rule of common but varying responsibility was P7. This enshrined the two major economic principles of polluters' pay, which was set as P15. It brought about the principles on the participation and the significance of particular groups for SD (P10, P20, P21 and P22).

About 2 decades later, there was a conference of the United Nations on SD (i.e. Rio +20) in June 2012. The conference was an agreement by the states to consider launching the procedures meant to develop SD goals and objectives, which are a fundamental tool for pursuing coherent and focused actions on SD. Other significant frameworks e.g. Millenium development goals, were to demonstrate that leaders of the world can collaborate to focus on the major problems in the global e.g. financial and war issues, which also includes poverty. The major variation between the Millenium development goals and the SD goals is the dimension of agreement, which is present amount the nations on the broad prevailing objectives, which includes the contexts of Millenium development goals and SD goals. These goals are a prevailing of collective action issues that are a central focus on SD, and the normal failure of nations at mitigating these issues.

The major policy dimensions and goals of SD were draw from the high-level panel of international sustainability from United Nations in 2012 [3]. This is utilized to establish consistent and uniform principle review on any framework about SD. The major policy dimensions and goals of SD stipulate that has to be universal in character, which covers the countries other than developing nations. Secondly, it has to express widely acknowledged globalized approach for SD. It has to incorporate a wider key area, which is not completely covered in Millenium development goals. It has to be comprehensive that reflects on 3D of SD. Thirdly, it has to incorporate benchmarking whereas being long-term in its context, focusing ahead to 2030. Fourthly, it has to include all the essential stakeholders in the mobilization and implementation of resources. Fifthly, it has to provide the overall score of analysis and review of goals based on the evolving scholastic evidence.

Contrary to that, during the current research, tasks have been identified as a collection of measureable indicators, which is also challenging. Due to the fact that indicators are elaborated beginning from the dimension of SD, there are no similar method among firms regarding the type and number of dimensions.

For $21^{\text {st }}$ agenda, article 8.6, nations could structure and develop frameworks and systems for evaluating and monitoring the progress towards the accomplishment of SD through the adoption of indicators. These were useful in evaluating measures in the environment, social dimensions and the economy. UNESCO considers 3D of SD following political dimensions (decision-making, politics and democracies) [4]. Cultural essentials play a vital role in public planning, which contributes significantly to cultural dimensions for SD, which considers that if community's culture disintegrates, everything will change. The actions of culture is essential to lay foundation for SD for the future generation focusing on the advancement of IE, power and electricity.

This paper section has introduced the concept of SD and IE and how the upcoming generation focusses on using them, including power and electricity. From this section, it is seen that the concept of SD was introduced earlier and principles designed for the same. The summit at Rio de Janeiro in 1992 established agreements that launched procedures of developing SD goals and objectives. Millenium development goals and SD goals were also established to focus on SD and IE. The remaining sections of the paper focus on completely evaluating the main aim of this research contribution, which is to discuss the theoretical assumptions of SD and Industrial Energy (IE). Therefore, the paper has been organized as follows: Section II provides the definition (pillars and dimensions) of SD and SE. Section III presents the literature review section. Section IV discusses sustainable development (SD) and sources of energy. Section V evaluates the consumption of IE. Section VI evaluates the pillars and dimensions of SD and IE. Finally, Section VI concludes the paper and provides future research directions.

\section{DEFINITION, DIMENSIONS AND PILLARS OF SD AND IE}

\section{A. Definition}

The theoretical assumption of IE is based on the projections and attempts to implant the fundamental principles of SD. The accessibility to IE is a fundamental element considered in the enhancement of civilization of modernized communities. Due to this, the sector of IE is one aspect that will be changed towards SD. The direction to which change happens is not typically normal. For this purpose, it is fundamental to explain what IE is for the sake of upcoming generations. Different from the appearances, it is not as unambiguous and clear as expected.

The precise definition of the term is however not clear. Presently, there is no loading explanation that explains Sustainable Energy (SE) and it is also evident that in most instances the definitions presented by researchers are different from each other based on designs. This is certainly evident in the cases of paraphrases of definitions of SD, which signifies the developments of the needs of the current generation that does not minimize the capacity of future generation's needs. The statement has not become a major basis that focusses on the primary concerns of permanence. 


\section{B. Dimensions and Pillars}

It is fundamental that availability is assured based on the prevailing needs of the ecosystem. Nonetheless, it is fundamental to note that the theoretical assumptions of SD incorporate three significant pillars. One of the pillars is community. Its availability is not as displayed as environmental problems, but appears in the explanations of industrial energy and SE.

1) Dimensions

This notion critically dominates in various works to define SE and policies under three critical dimensions: sociocultural, economic and ecological. In this manner, sustainability approaches adopted incorporate:

- Environmental dimension: Global warming, utilization of non-renewable resources, natural tolerance, overutilization of renewable resources, problems of the human wellbeing

- Economic dimensions: These represent the implication of the country's economy, which meets the requirements of IE, accomplishing the needs of SE, ensuring short-term privacy of supply, focusing on costeffectiveness and dependency on the supply of raw materials

- Socio-cultural dimensions: The terminologies social tolerance, perpetual supply assurance, incorporation with the present infrastructure, and eliminating participation in international issues are some of the dimensions considered

2) Pillars

The three-pillar categorization of sustainable energy is also given as a highlight of various methods meet by the consideration of energy in sustainability. The pillars considered in this case are:

- Ecological pillar: This pillar focusses on the implications of the use of certain technologies, which are a fundamental subject of ecological assessment. In that case, the evaluation has to be considered based on the volume by the implications of energy and environment. Tools such as the analysis can be focused on complete fuel cycles and life cycles. This is possible using the application of thermodynamic processes and analyses of ecological life cycles.

- Economic pillar: This has to be analyzed based of the ecological cost-benefit evaluation.

- Social pillar: This pillar is more difficult to utilize the measureable approach; for instance, through the level of inclusion of relevant stakeholders, mapping, preferences and customer research. Contrary to that, it is advisable to utilize ethical evaluation of the implications of the present and future IE system on the community and environment. One major aspect of this evaluation is the issue of inter-generational equity based on the accessibility of energy sources and the acquisition costs.

\section{LITERATURE REVIEW}

A. Maulud and H. Saidi in [5] argued out the balance between personal energy initiatives and characterized them based on large error margins, which result from the partaking of initial assumptions. The resultant evaluation of the sources varies even by $20 \%$. This incorporates not only the application of effective weights assigned to particular factors, but their choices as well. Moreover, these maps can vary due to the selection of the incorporated measures and approaches. J. Káposzta in [6] introduced some of the approaches to sustainable development.

L. Srivastava in [7] evaluated environmental approach for climate protection. The amount of greenhouse gas in every $\mathrm{kWh}$ was considered. The tolerance limit of nature based on the usage of surfaces per $\mathrm{kWh}$, which is the effect on protected species. Another approach is the usage of resources where the consumption of non-renewable consumption of resources is considered in every $\mathrm{kWh}$. Another approach considered in the health threat where harmful emission, hazardous wastes and radiations have been considered in every $\mathrm{kWh}$.

P. van Griethuysen in [8] evaluated the economic approach of sustainable development. With this approach, the effect of the economy was felt whereby tasks in every $\mathrm{kWh}$, trades' turnovers of industries have been considered. Another factor being considered is the certainty of the supplier in short-term industrial applications. Another key factor considered is the appropriate price, which include concentration and profitability, where costs in every $\mathrm{kWh}$ is considered. Contributions to the economic independence focusing on the supply of raw materials have been considered. The last consideration in the economic approach is technician efficacy, which signifies energy depreciation and efficiency.

P. Virtanen, S. Saarinen and M. Kamppinen in [9] evaluated the socio-cultural approach where socio tolerance aspects, which evaluate the acceptance of people, have been considered. Another aspect is the long-term privacy and 
security concerns of supplies, which include energy sufficiency and its potentials. The third aspect is the capacity to incorporate the existing structures and the fourth one is the global conflicts and its prevention measures.

Out of the above evaluated approaches, security is one of the most critical aspect with some specified measures. These measures have led to various interpretations of results that have been obtained based on the application of this kit. Moreover, some of the critical indicators may raise the doubts in the evaluation of the application in a particular country. I. Manners in [10] argues that some indicators may potentially be challenging hence leading to global conflict preventions. Nations with significant coal research utilize them to power, electricity and energy production, withdraws from international markets, and hence contributes to the reduction of upward pressure on the demands and pricing of substitutes e.g. natural gas.

Y. Hu [11] comments that energy products e.g. coal can be imported into countries and can be affected by political tension in cases of inadequate supplies. This same concern may be raise renewable sources of energy. In that case, it is easier to recall the disputes and issues between Uzbekistan and Tajikistan on the usage of reservoirs. The first of these nations, through the establishment of hydroelectric energy stations on reservoirs can become power dependent from their neighboring countries.

Nonetheless, the establishment of dams and other reservoirs can minimize the chances to access waster in neighboring countries, in this case Uzbekistan. Diplomatic issues and disputes have been witnessed for many years and its end it not evident any time soon. V. Bondarenko in [12] commented that hydroelectric power, which should not affect global tension, has significant impact on global relations in areas were water is considered a problem.

B. Bossink in [13] has therefore presented a definition of SE, which signifies the collaboration of local governments ambitious in supporting the dimensions, approaches and pillars of SD. In reference to the representatives of SE, negative functioning of natural schemes and models can influence the negative implications on human health. The main purpose of this is to ensure sustainable security for the current and future generations. It is to be attained through the interlinkage of savings, efficient solutions, and efficient technologies, include the application of renewable energy.

B. Bossink in [14] considers the wider definition that captures the concept since it displays not only the problem of permanence, but also show that it is a source of energy altering the health of humans and the environment. This form of provision is significant since, practically; there are no sources of energy affecting or influencing damages to the ecosystem. This explanation indicates that in various cases, SE is regarded not a single factor of energy source, which permits reinforcement of particular actions e.g. power and temperature, but also the complete system hence allowing the obtaining, transportation and transfer of SE to consumers.

M. Centeno Brito, K. Lobato, P. Nunes and F. Serra [15] argue about the differentiation between SE and SE systems. However, the distinctions are considered pointless since, in many cases, SE policies are considered. The theoretical assumptions of SE and IE are considered essential in the formation of SE systems, which are projected to meet the requirements of the concept compared to acquisition sources, because the issues about the efficient sustainable energy management are as fundamental as its production.

\section{SUSTAINABLE DEVELOPMENT (SD) ENERGY SOURCES}

To establishment SE, energy sources have to considered. These are the energy sources, which are not significantly depleted by continuous application. The application of sources, which does not affect the emission of pollutants and other dangerous substance to the ecosystem on a large scale, is considered. The application of sources does not include the consolidation of critical threats to social injustice and health. Irrespective of the high generality of the present approach evaluated in this research, it is still challenging to correctly identify sources of energy, which meet the demands of users $100 \%$. Due to this reason, the concept of primary SE is relative since the same source may be sustainable. The variation in approach stems from the deficiency of unanimity within the concepts of SD.

The discussions of the necessity of applying SD is based on both its weak and strong form, which is essential because of the existence of four major forums of sustainability standards. Resultantly, it is challenging to project unanimity in SE systems. This is because it would nearly be impossible to establish restrictive forms of sustainability (due to insufficiency of systems and sources and the theoretical assumption that the economy would need to undergo significant transition). Weak sustainability has to be handled as a dominant form sharing during the period of transition.

Economic enhancement without transition in coverage or the condition of SE flow is not possible because energy is a major unit in the physical world. Due to this reason, it is significant to the globe and its transition in SE flow incorporates ecological implications. For this purpose, the decisions about the acquisition of SE are connected to the compromises. It is fundamental to choose the remedy that will be connected to a significant development, such as enhancing efficiency, and the one that will cause minor damage to the environment. Identification of strong sustainability as a leading remedy for SE implies that only the renewable sources can be termed as sustainable. This is majorly because of their renewability, which shows their relevance in the future. 
In this manner, the durability is the main concern here. Renewable energy impact relatively to the ecosystem and the synonymous application of renewable and sustainable energy is considered. Renewable sources, mostly on a local scale can result in negative effects, which is destructive for the entire concept. For instance, the provision of $100 \%$ energy to a local, isolated energy system with just a single source e.g. winds energy. In case adverse weather conditions eg the installation, will not provide power, energy and electricity and thus considered ineffective. Another critical example of ineffective application of renewable IE can be the formation of larger installations that utilize biofuels, which is the process capacity making it essential to source raw materials from distances.

In drastic condition, IE value of loads can exceed the expense of energy utilized for transportation. In this manner, irrespective of the fact that the ecological costs of emissions (plant) continue to meet higher standards, violations occur at social aspects of SD. Lastly, the theoretical assumption of SE concerns the permanent provision of human beings. For this purpose, sustainable renewable IE sources are those that are inexhaustible and less harmful to the ecosystem, climatefriendly and socially acceptable. Partially unpredictable and variability characters of renewable IE, including storage issues (mostly in the case of electricity) hence make it challenging to implement it in IE systems.

For this purpose, spatial planning is critical in the building process of SE. However, it incorporates various aspects: it leads to reduction in SE demands; it has to incorporate the segments of enhanced pre-disposition of IE conversion e.g. the segments of windmills and solar panels. The third aspect is the enhancement of energy plants, which considers the local capability to produce IE not over-extending the distances of transportation (certainly in biomass). The fourth aspect is coordination of spatial planning and IE, which permits optimal application of IE sources and the transmission of networks.

Another example of ineffective application of renewable sources is the application of complete-value timber. Such consumption, mostly in developing countries, on a smaller scale can be justified (mostly in the absence of other intuitive alternatives). However, the usage of wood for fuel in larger boilers to meet legal standards, eg in Great Britain and Poland, has to be considered not just as irrational, but hazardous to the ecosystem and human beings. It has to be considered that the usage of wood wastes is not doubted. These incorporate public initiatives such as the combustion of trees.

In case the trees are not needed at home, it is better to utilize them in industrial furnace compared to simply leaving to heap. In addition, these actions and its results are normally publicized hence amounting in attention regarding the problems in the environment and energy consumption. Practically, there is no doubt that renewable sources of energy inclusively are some of the technologies of sustainable initiatives. However, their application has to consider some factors, incorporating capacities, accessibility to sources, energy demands in areas and dispersion sizes. It is fundamental to consider that the retrieval of IE from renewable sources of energy affects ecological costs. Due to this, in some literature assumptions, it is noted that none of these sources are identified by human beings in the modern age, and are not considered sustainable in any way.

Nonetheless, this ideology is to be counter-intuitive based on incentives designed to obtained novel and enhanced remedies. It is challenging to image the condition in which all the IE would originate from e.g. renewable sources. This is because of various factors: The major barriers are limited human capacities for the storage of energy, higher costs, and lack of globalization to mass production and installations, including the lack of political will. Environmental circle has been dominated by the ideology that humans require to apply restrictive standards for sustainability i.e. renewable IE.

In some developing countries, non-renewable technologies are also considered as sustainable. Particularly, this critically concerns the usage of nuclear energy and hydro energy. The attitude is mostly noticeable in UK, France and USA where there is significant tendency to consider nuclear energy in sustainability. In addition, it considered that because of limited capacity of humans to obtain bulk energy quantities of energy from renewable energy sources, it is vital to consider SD schemes based on fossil fuels. According to G. Midgley and M. Reynolds in [16], SD schemes are considered as creating the right measures of energy. This is the best example as forming the right mix of energy developing approach, which considers the measures to enhance the efficiency of energy whereas enhancing the share of gas. In this manner, it is possible to attain reduction of ecological implication.

Nonetheless, it has to be assumed that the approach is only temporary, and might lead to more reductions in emissions, which can be considered as a move far from the gas as primary energy source. Irrespective of the inclusion of gas to the strategy of building SE schemes, nobody will treat this as a suitable source. A slight varying approach is noticeable for the application of the atom, where there is a firm division between opponents and supporters of this approach of producing energy. Discussion in the groups not only considers the theoretical assumptions, but also translates into the policies of many developing countries. In that regard, Denmark and Austria are considered as the enemies leading to the discontinuity of the expanding nuclear energy and the slowly closing reactors.

The same decision has been made in Japan due to the Fukushima disaster. Contrary to that, as an example, one can present France, where nuclear energy accounts for approximately $40 \%$ of the consumption of primary energy. Similar positive attitude over this form of technology is apparent in South Africa, South Korea and China. Moreover, more countries have focussed on building novel energy plants irrespective of moderate social acknowledgement for such initiatives. This incorporates Poland, Finland, and Czech Republic. More positive attitude towards nuclear energy stems from the beliefs of relatively lower hazards in the ecosystem of the plant. In that case, it normally focus on $0 \%$ emission of carbon dioxide to the atmosphere in the process of acquiring energy hence amounting to the reduction of energy impact to the formation of global warming. 
Cost levels on the other hand are not fundamentally smaller. A major issue associated with nuclear energy is still a prevailing concern of nuclear wastes and it possess significant threat of ecological contamination. In the context of SE, it is normally mentioned about novel technologies that are not completely explored. They may be critical for the development of the future. The most frequently mentioned technologies is structuring of thermonuclear reactors and usage of hydrogen. Nonetheless, it is important to consider that both invention implementation and dissemination of innovations take a long time. In case of hydrogen and incorporated fuel cell initiatives, the qualification of clean technologies is linked to fuel generation technologies. In conditions where it generated in renewable approaches, it has to be justified as a source of sustainability.

Based on the above arguments, it is problematic to determine which sources of energy should be considered as sustainable. In addition, such studies have to be placed in particular contexts, where co-existence of various technologies has to be evaluated based on the prevailing conditions. They have to consider the emission caused by IE and its conversion procedures, but also in the transportation of primary IE. The initiatives based on the combustion of natural gas can be hazardous as burning coal especially when they are recorded transmission losses because of bad pipeline conditions. In reference to thermodynamics laws e.g. the rights of entropy, identifying $100 \%$ sustainability of IE seems to be challenging. Due to this, the evaluation of energy and its transformation in SD has to be utilized as a more flexible method. This is because sustainability has to be considered a critical source with longer life cycle and permit ecological implication.

\section{CONSUMPTION OF INDUSTRIAL ENERGY}

One of the fundamental terminologies linked to the theoretical assumptions of SD is the demand for dematerialization of energy consumption. Its major objective is to consider reducing the present pressure on the ecosystem resulting to the necessity to have more good, thus enhancing the demands for natural resources. This issue is also fundamental in modern IE sector where one of the most significant problems is about security of supply for non-renewable energy resources. This issue attracts the attention of economists, but also the politicians. Evidences of this incorporate recognition of IE as a single factor in the some series of reports, which are global trends.

Particularly, it relates to oil and peak oil. It considers the momentum when one can extract maximum possible amount of resources and raw materials from the actual field. Later on, productivity will drop because of technical barriers. This is the first and most fundamental warning signal exhaustion of the energy sources. The efficiency of industrial energy has to be the basis of policy formulation of all nations. This is certainly fundamental in the absence of the possibility of global usage of inexhaustible and ecologically friendly IE technologies. One of the major pillars of this action is to minimize the major IE demands by enhancing the efficiency of devices.

Moreover, the efficiency of IE amounts to the minimization of dependency on imported fossil fuels and minimize pollution. It is fundamental to remember that effectiveness is not a remedy, but a possibility extension of utilizing the present methods of acquiring final IE. The most general and modest way to define energy efficiency is minimizing energy amount essential in performing particular tasks (for instance, produce the services and products). The methodology is also in the European Union where it is considered as the ratio between an output of IE, goods and services, including energy inputs.

A. Lichtenberg in [17] has highlighted the major variation between energy savings and energy efficiency. Energy savings is a terminology that defines the amount of saved IE determined by evaluating or estimating consumption after and after implementation of energy efficacy improvement approaches whereas guaranteeing normalization of external factors affecting the consumption of energy. Reduction of energy consumption can be achieved through the application of effective remedies, and through energy savings. The second approach relates to final consumers (businesses and households) leading to the minimization of demands for goods and services.

The processes of efficiency can be applied in the conversion of energy, consumption and transmission. Systems with more efficiency e.g. generators or car engines can contribute fundamentally to the minimization in energy consumption. Nonetheless, the major advantage based on performance can be witnessed in the approaches of cogeneration, which is the simultaneous production of heat and electricity. The same way, attention to construction and quality of transmission lines and dispersed production schemes based on localized energy sources can enhance efficiency.

End users are a major factor of efficiency since energy consumers select particular technologies. This is applicable to lines of production and purchasing of household appliances, such as refrigerators and washing machines. It is fundamental to note that the costs relate significantly to consumers in Small and Medium-Sized Enterprises (SMEs) and households. The entities will be stimulated to purchase new and effective equipment and consume less energy.

Nonetheless, these actions are possible with higher energy prices, which formulate the incentives meant at replacing the present devices with efficient ones. Normally, the products consuming less IE are costly resulting from technological advancement and product marketing. The choice to purchase made by consumers has to be justified by future advantages, whereby the savings from energy reduction and consumption. Nonetheless, it has to be noticeable to influence the purchasing of devices. Due to this, high prices of energy are a fundamental stimulus of energy efficacy. Nonetheless, contrary to that, it is fundamental to not that SD implies meeting the needs of the current generation.

In case of IE, it is considered as shaping of prices of goods to avoid making it a barrier for consumers when it comes to affordability. This shows the necessity for a cautious methodology and strategy to pricing as a factor of growth of 
efficiency. Based on the above assumptions, the end users are a major agent facilitating change in the efficiency of energy. Nonetheless, in most instances, actions result from the factors, which are far from economic incentives. This is because of the challenges of precise assessment of efficiency.

For this purpose, regulations by the states are fundamental and this shows and force trend. Experiences over the past few decades indicate that most of the globes largest economies have been undertaken based on the initiatives that intensity the activities of energy efficiency and its activities. It is projected that in 2010, a global effect of minimizing the consumption of energy by approximately $35 \%$ was felt. In an attempt to assess efficiency problems, it is fundamental to consider Jevons paradox. Enhancing the efficiency of coal usage leads to a declining price and therefore enhances the consumption of energy.

Finally, the increase in the efficiency of machines is assured using various forms of primary energy consumption measures. The observations from about a century ago have confirmed the current research on different countries in America and Europe. This shows that the development of efficiency is not just apparently a fundamental tool for attaining sustainable IE, but also an approach for the usage of energy-saving resources. Consumers in developing countries display their ambition to save energy, but also utilize more devices in the process. Due to this, numerous forecasts for energy consumption show further development and growth.

\section{THE PILLARS AND DIMENSION}

The theoretical assumption of SD is based on three fundamental pillars: social, environmental and economic development. The evaluation of energy and its efficiency typically concentrate on ecological aspects, which also consider issues about the economy. The third pillar has been ignored or rather marginalized. The result is that numerous energy policies consider the ecological issues as a climate policy instead of a sustainability policy. The significance of energy for the enhancement of civilization is evident.

Nonetheless, about 1 billion people have limited accessibility to electricity while the same number of people utilize unsafe network. Moreover, approximately 3 billion individual utilize biomass to provide heat energy and prepare food. This shows that more than $40 \%$ of the population faces problems with the accessibility of energy. This segment has to be regarded and eliminated from the path of SD. The global economy considers the initiatives to transform this condition, but it is considered that, irrespective the projects to grant them full ranking rights, the development objectives are unsatisfactory.

In this manner, provided that IE is one of the major factors of SD, social aspects of SE have to be a major concern of this theoretical assumption. Nonetheless, we can visualize the opposite of the tendency, i.e. those actions that exclude critical issues. Initiatives have to be implemented, including the technologies about renewable energy considering the needs of the society. In that regard, attention is dedicated to the measures to minimize the developments of unclean energy technologies in developing nations. This is defined based on the necessity to serve the wellbeing of the globe.

Typically, it results that greenhouse gases and its emissions are significantly lower in developing countries compared to developed ones. This implies focusing on regulating the globe without the rules such as justice. It can be considered that the principles focused on establishing high-quality ecological standards result in high-energy costs in developed countries. It is fundamental that in these segments, there are groups of people with less capacity to afford unrestricted usage of IE. The trend can be visualized in countries such as UK and Germany, which are developed in terms of renewable sources of energy and markets in the globe.

Another key issue connected with the usage of renewable sources of energy is massive voltage fluctuation in mains. In most instance, e.g. in SMEs, this issue can lead in significant effects because of the shut-offs of more sensitive automated equipment e.g. computers and machinery. Linking to the network, numerous renewable power plants enhance the risks of failure because energy can no longer be stored. This shows that during times of high production i.e. during stronger and proper winds, there is more energy that has to be utilized. This issue can be noticed everywhere in the globe. For instance, in central Europe, this has stimulated political issues.

Due to more wind energy in developed countries such as Germany, problems have been caused in not only the country, but also those neighbouring it e.g. Poland and Czech Republic. Over the past few decades, a clear trend can be visualized to reform the energy markets e.g. the shifts from monopoly to higher potential competitiveness and marketability. The initiatives has amounted in the need for competitive pricing in sectors of intermediaries and producers. This has resulted in the accessibility of affordable energy. Nonetheless, these actions may cause some negative effects. The first one is previously mentioned limiting of propensity to save IE and dispense it at a cheaper price.

Another effect is the lack of investments in novel technologies and networks of transmission. This is seen in Europe, whereby up to 2030, it is projected to replace nearly a third of the capacity to product IE and considering approximately 30 , 000 lines of transmission. Restrictions of investments apply to not only modern infrastructure but also limits innovations, enhance efficiency, and minimize environmental hazards from the conversion of energy and its installations. One of the best examples in this case is the European market, where irrespective the number of initiates, the emission trading and electricity production expenses are higher compared to marketing prices causing minimal interests in the market. 
It is considered that because of the condition in the future, Europe will pressurize the member states to rectify emissions of trade systems, which manually enhance the pricing of permits. These actions imply that the evaluation of SE has to concentrate on market and social problems not just environmental issues. The processes of enhancing the efficiency of energy are considered slower over the decades. The result is that one should never expect rapid changes in technological application. Factors, which may cause significant changes, include markets and social awareness, and these factors are capable of promoting reliable skills concerning the threats and opportunities about particular technologies instead of forming administrative guidelines.

Many problems are linked to the market that in one hand has to be open as potential and with low barriers to entry and can also be controlled in the segment hence allowing both affordability of implementing novel technologies and ensuring low pricing hence facilitating accessibility of the end user. An example of failure of the market is minimal interests in technological application, storage and C02 capturing since these can be implemented widely in larger firms whose management is obliged to attract profits. Applying the technologies is acknowledged from the ecological perspective, but considered economically unprofitable up to a decade later. It is thus challenging to project that these decisions will be formulated exclusively based on market-based remedies. Contrary to that, CCS renewable SE is handled as a single foundational tool in facilitating SD and IE in the future generations.

\section{CONCLUSION AND FUTURE DIRECTIONS}

This paper has analysed SD as a concept that is formulated to provide the globe with the opportunity to structure longterm suitability of IE, power and electricity. One of the major pillars of modern advancement is IE. Due to this, it is fundamental to concentrate widely on the subject matter. Concentrating concepts on two critical pillars i.e. renewable IE and enhancing efficiency and minimization of energy consumption are a major way of defining sustainable energy. Nonetheless, this approach provides an incomplete image of this critical concept due to the fact that is does not consider markets that are a major factor that determine energy availability. Considering the pillars evaluated in this paper, SD stresses that energy has to be considered widely i.e. based on processing, consumptions, distribution and transportation. Due to this, based on the concept of SE, the complete energy scheme is considered. The dimension of this implication will vary depending on local markets to international relations. Nonetheless, in most instance, it should permit the end users to provide IE. The above discussions and theoretical assumptions of SD and IE are based on defining the concepts of energy. However, the research is limited to concepts that should be focussed on future research: differentiating sustainable and unsustainable concepts; evaluating the chances of operationalization, which defines the indicators that evaluate and determine sustainability. Critical analysis of applicable SE policies since it is considered that SE has to be considered as a scheme of consumption, distribution, transportation and processing of IE. This has been characterized by overwhelming and constant reduction of the use of non-renewable energy sources.

\section{References}

[1]. L. Taylor, "Sustainable development: An introduction", World Development, vol. 24, no. 2, pp. 215-225, 1996. Available: 10.1016/0305750x(95)00130-5.

[2]. S. Benatar, "The poverty of the concept of 'poverty eradication'", South African Medical Journal, vol. 106, no. 1, p. 16, 2015. Available: 10.7196/samj.2016.v106i1.10417.

[3]. M. Watanabe, "The United Nations Sustainable Development Goals", BioScience, vol. 70, no. 3, pp. 205-212, 2020. Available: 10.1093/biosci/biz155.

[4]. M. Burri, "Advancing the Goals of the 2005 UNESCO Convention on the Diversity of Cultural Expressions in the Fields of Education, Participation of Civil Society and Sustainable Development in the Digital Age", SSRN Electronic Journal, 2013. Available: $10.2139 /$ ssrn.2364956.

[5]. A. Maulud and H. Saidi, "The Malaysian Fifth Fuel Policy: Re-strategising the Malaysian Renewable Energy Initiatives", Energy Policy, vol. 48, pp. 88-92, 2012. Available: 10.1016/j.enpol.2012.06.023.

[6]. J. Káposzta, "New approaches to achieve sustainable regional development", Visegrad Journal on Bioeconomy and Sustainable Development, vol. 2, no. 2, pp. 50-52, 2013. Available: 10.2478/vjbsd-2013-0009.

[7]. L. Srivastava, "Climate protection for sustainable development or sustainable development for climate protection? A case study from India", Global Environmental Change, vol. 16, no. 2, pp. 120-122, 2006. Available: 10.1016/j.gloenvcha.2006.01.005.

[8]. P. van Griethuysen, "Sustainable development: an evolutionary economic approach", Sustainable Development, vol. 10, no. 1, pp. 1-11, 2002. Available: $10.1002 /$ sd.175.

[9]. P. Virtanen, S. Saarinen and M. Kamppinen, "How to integrate socio-cultural dimensions into sustainable development: Amazonian case studies", International Journal of Sustainable Society, vol. 4, no. 3, p. 226, 2012. Available: 10.1504/ijssoc.2012.047279.

[10]. I. Manners, "Assessing the decennial, reassessing the global: Understanding European Union normative power in global politics", Cooperation and Conflict, vol. 48, no. 2, pp. 304-329, 2013. Available: 10.1177/0010836713485389.

[11]. Y. Hu, "Tension term, interchange symmetry, and the analogy of energy and tension laws of the AdS soliton solution", Journal of High Energy Physics, vol. 2009, no. 05, pp. 096-096, 2009. Available: 10.1088/1126-6708/2009/05/096.

[12]. V. Bondarenko, "Dual-Delivery Turbine Scrolls in Large Hydroelectric Units of Underground Hydroelectric Power Plants", Power Technology and Engineering, vol. 51, no. 4, pp. 410-413, 2017. Available: 10.1007/s10749-017-0847-3.

[13]. B. Bossink, "Demonstrating sustainable energy: A review based model of sustainable energy demonstration projects", Renewable and Sustainable Energy Reviews, vol. 77, pp. 1349-1362, 2017. Available: 10.1016/j.rser.2017.02.002.

[14]. B. Bossink, "Learning strategies in sustainable energy demonstration projects: What organizations learn from sustainable energy demonstrations", Renewable and Sustainable Energy Reviews, vol. 131, p. 110025, 2020. Available: 10.1016/j.rser.2020.110025.

[15]. M. Centeno Brito, K. Lobato, P. Nunes and F. Serra, "Sustainable energy systems in an imaginary island", Renewable and Sustainable Energy Reviews, vol. 37, pp. 229-242, 2014. Available: 10.1016/j.rser.2014.05.008.

[16]. G. Midgley and M. Reynolds, "Systems/operational research and sustainable development: towards a new agenda", Sustainable Development, vol. 12, no. 1, pp. 56-64, 2003. Available: 10.1002/sd.218.

[17]. A. Lichtenberg, "Energy efficiency and energy savings", Energy, vol. 2, no. 3, pp. 283-286, 1977. Available: 10.1016/0360-5442(77)90032-9. 